\title{
Expression of 14-3-3 $\zeta$ and interaction with protein kinase $C$ in the rat retina in early diabetes
}

Received: 11 October 2004 / Accepted: 23 February 2005 / Published online: 21 May 2005

(C) Springer-Verlag 2005

\begin{abstract}
Aims/hypothesis: The present study aimed to investigate the expression levels of and the relationship between 14-3-3 $\zeta$ and protein kinase C (PKC) in the retina of early diabetes. Methods: Changes in the expression levels of, and interaction between, 14-3-3 $\zeta$ and PKC were investigated by Northern and Western blot analyses, immunoprecipitation and double immunostaining in the retina of diabetic rats after 6 weeks of diabetes. PKC activity was examined using a PKC assay. Results: In the diabetic retina, the molecular levels of 14-3-3 $\zeta$ were reduced, while those of PKC $\beta$ and $\zeta$ were increased. Direct interaction between 14-3-3 $\zeta$ and PKC was markedly decreased in the retina after 6 weeks of diabetes, while PKC activity was increased. Conclusions/interpretation: These findings show that a reduction in 14-3-3 $\zeta$ can induce PKC activation, suggesting that this is a main cause of visual dysfunction in the retina during diabetes.
\end{abstract}

Keywords $14-3-3 \zeta$. Diabetes $\cdot$ PKC activation $\cdot$ Retina Streptozotocin

\section{Abbreviations PKC: Protein kinase C}

\author{
Y. H. Kim · Y. S. Kim · S. S. Kang · H. S. Noh · H. J. Kim \\ G. J. Cho · W. S. Choi \\ Department of Anatomy and Neurobiology, \\ College of Medicine, \\ Institute of Health Science, \\ Gyeongsang National University, \\ Jinju, South Korea \\ W. S. Choi $(\bowtie)$ \\ Department of Anatomy and Neurobiology, \\ College of Medicine, \\ Gyeongsang National University, \\ Chilam-dong 92, \\ Jinju, 660-751, Kyungnam, South Korea \\ e-mail: choiws@nongae.gsnu.ac.kr \\ Tel.: +82-55-7518716 \\ Fax: +82-55-7590779
}

\section{Introduction}

The isotype 14-3-3 $\zeta$, along with others, has been reported to play a critical role in the central nervous system [1-4], including in neuronal differentiation [5] and synaptic plasticity [6]. Moreover, this isotype is present in significant amounts in the retina, as well as in the brain, and is necessary for light adaptation processes or differentiation in photoreceptors [7, 8]. It is well established that 14-3-3 proteins function as a protein kinase $\mathrm{C}$ (PKC) regulator [913]. In vitro, 14-3-3 $\zeta$ has been reported to be an endogenous PKC inhibitor [5, 14], but the role of $14-3-3 \zeta$ in the diabetic retina remains unclear.

PKC has been implicated as an important mediator of diabetes-induced vascular dysfunction [15-17]. Furthermore, it is well known that activation of PKC in the diabetic retina contributes to abnormal haemodynamics and blood flow [18-20].

In this study, we investigated these interactions as well as changes in molecular levels to understand the significance of the relationship between 14-3-3 $\zeta$ and PKC isoenzymes in the retina of early diabetes.

\section{Materials and methods}

Animals Diabetes was induced in male Sprague-Dawley rats (250-300 g; Samtako, Osan, Korea) by streptozotocin (50 mg/kg; Sigma, St. Louis, MO, USA). After 6 weeks of diabetes, rats were used as previously described [21]. Diabetes was defined as blood glucose being above $13.9 \mathrm{mmol} / \mathrm{l}[21]$.

Northern blot analysis Total retinal RNA was isolated using TRIzol reagent (Gibco BRL, Gaithersburg, MD, USA) according to the manufacturer's instructions. Retinas from ten control and ten diabetic rats were pooled, and $20 \mu \mathrm{g}$ of total RNA from each group was used for testing. All procedures have been described elsewhere 
a
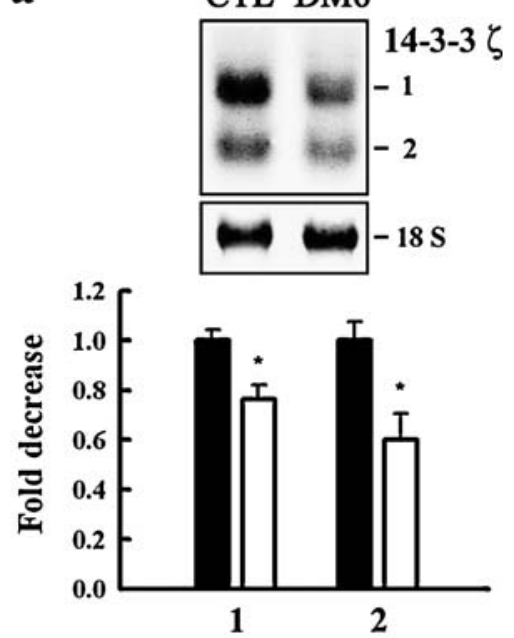

b

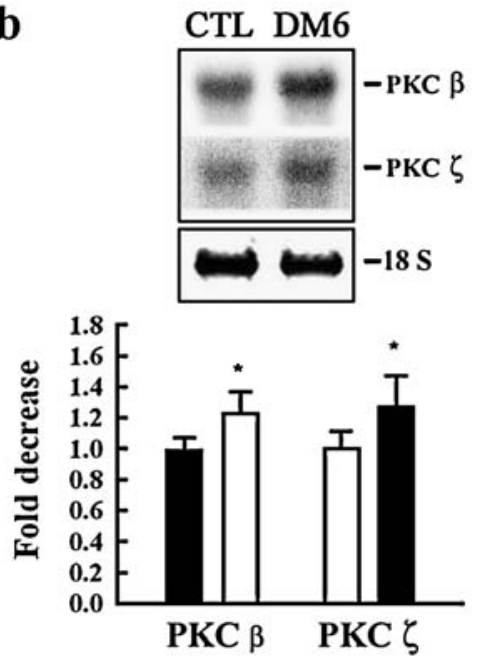

Fig. 1 14-3-3 $\zeta$ and PKC $\beta$ and $\zeta$ mRNA transcripts were decreased and increased (a and $\mathbf{b}$, respectively) in the retina after 6 weeks of diabetes. mRNA: 18S rRNA ratios are presented as a fold change in diabetes (white bars) vs control (black bars). CTL, control; DM6, after 6 weeks of diabetes; 1 and 2, 2.3 and $1.8 \mathrm{~kb}$ mRNA transcripts of $14-3-3 \zeta ; 18 \mathrm{~S}, 18 \mathrm{~S}$ ribosomal RNA as a loading control. Data are means $\pm \mathrm{SD}, n=3 .{ }^{*} p<0.05$ vs control

[21]. cDNA probes encoding 14-3-3 $\zeta$ and PKC $\beta$ and $\zeta$ labelled with $\left[\alpha-{ }^{32} \mathrm{P}\right]$ deoxycytidine 5 'triphosphate were used. Each specific band was normalised to $18 \mathrm{~S}$ rRNA for densitometry.

Primary antibodies Rabbit polyclonal anti-14-3-3 $\zeta$ antibody and mouse monoclonal PKC $\beta$ and $\zeta$ antibodies were obtained from Santa Cruz Biotechnology (Santa Cruz, CA, USA). Mouse monoclonal anti-PKC and $\alpha$-tubulin antibodies were from Sigma.

Western blot analysis Retinal proteins $(30 \mu \mathrm{g})$ were subjected to $10 \%$ SDS-PAGE and Western blotting using primary antibodies as previously described $[21,22]$. Specific bands were visualised by enhanced chemiluminescence and revised to the level of $\alpha$-tubulin protein for densitometry.

Immunoprecipitation Retinas were lysed in ice-cold radioimmunoprecipitation assay buffer $(50 \mathrm{mmol} / \mathrm{l}$ Tris $-\mathrm{HCl}$ [pH 8.0$], 150 \mathrm{mmol} / 1 \mathrm{NaCl}, 0.1 \%$ SDS, $0.5 \%$ sodium deoxycholate, $1 \%$ Nonidet P-40) containing several protease inhibitors. The lysates $(500 \mu \mathrm{g})$ were pre-cleared using protein $\mathrm{G}$ agarose (Santa Cruz Biotechnology) and immunoprecipitated with anti-14-3-3 $\zeta$ or -PKC antibodies. The immune complex was subjected to SDS-PAGE and blotting. Reciprocal experiments were performed to provide additional evidence.

PKC assay PKC activity was determined in $14-3-3 \zeta$ or PKC immunoprecipitate using a SignaTECT PKC Assay System (Promega, Madison, WI, USA) according to the manufacturer's protocol. The biotinylated ${ }^{32} \mathrm{P}$-labelled substrates were recovered from the reaction mixture using a

a
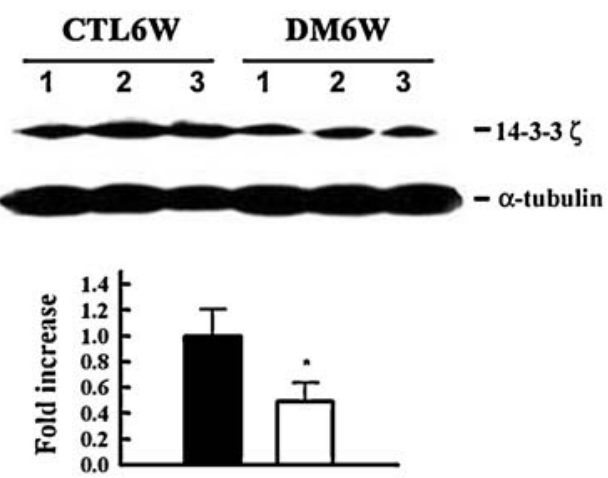

b
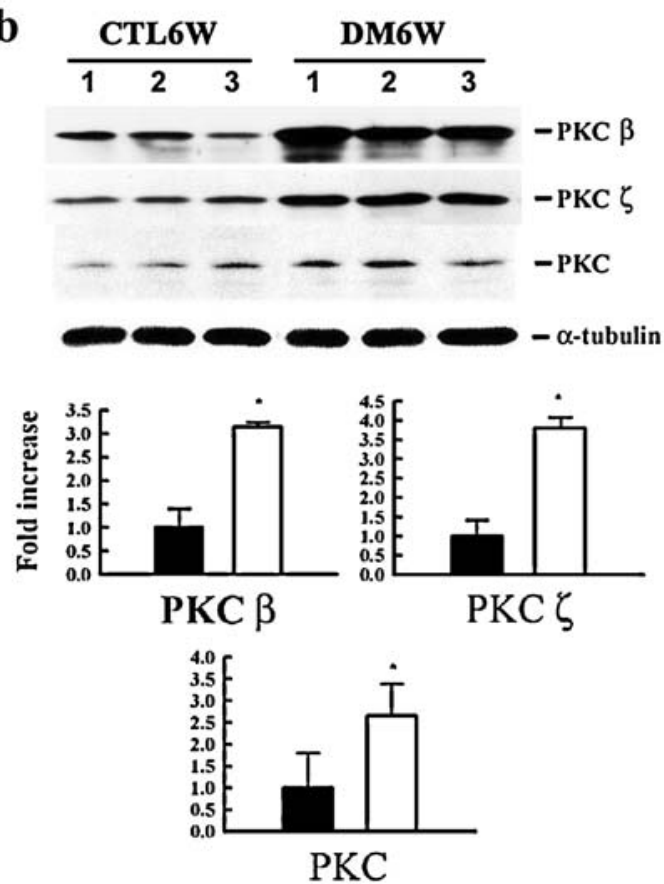

Fig. 2 14-3-3 $\zeta$ protein levels decreased in the retina after 6 weeks of diabetes (a), while PKC protein levels increased (b). Protein levels are presented as a fold change after normalisation by $\alpha$ tubulin in diabetes (white bars) vs control (black bars). CTL6W, control after 6 weeks; DM6W, after 6 weeks of diabetes. Data are means $\pm \mathrm{SD}, n=5 .{ }^{*} p<0.05$ vs control 
a

IP: anti 14-3-3 ל/ WB: anti PKCs

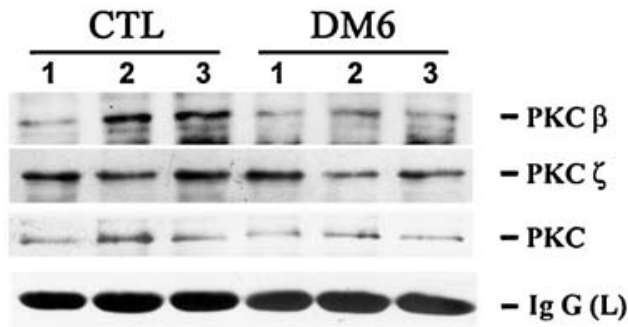

b

IP: anti PKC $\beta$ /WB: anti-14-3-3 $\zeta$

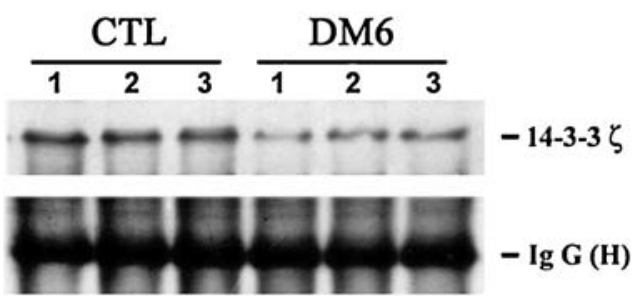

c

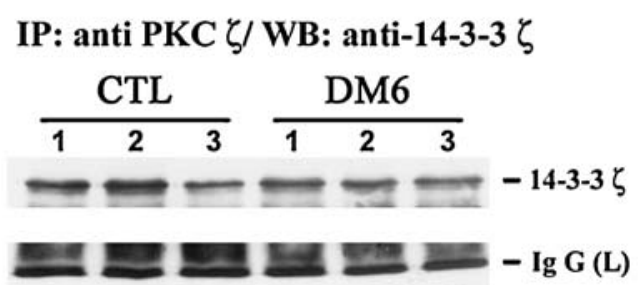

d

IP: anti PKC/ WB: anti-14-3-3 $\zeta$

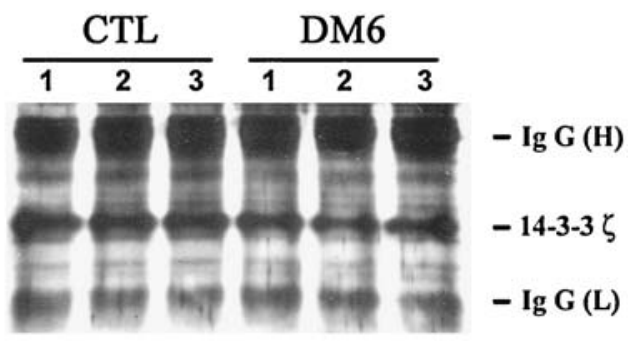

Fig. 3 Interaction between14-3-3 $\zeta$ and PKCs was reduced in the retina after 6 weeks of diabetes $(\mathbf{a}-\mathbf{d})$. The $500 \mu \mathrm{g}$ of retinal proteins was subjected to immunoprecipitation using the antibodies indicated, followed by Western blot analysis $(n=3)$. CTL, control; DM6, after 6 weeks of diabetes; $I P$, immunoprecipitation; $W B$, western blotting

$\mathrm{SAM}^{2}$ Biotin Capture Membrane (Promega). Alternatively, bound radioactivity was read using a scintillation counter.

Double immunostaining Retinal tissue preparation and immunofluorescence staining were carried out as previously described [22]. Sagittal sections $(12 \mu \mathrm{m})$ were incubated overnight in a mixture of total PKC and 14-3-3 $\zeta$ antibodies, followed by incubation in a mixture of two secondary antibodies: goat anti-mouse IgG conjugated to carboxymethylindocyanine, and streptavidin conjugated to $\mathrm{Cy} 2$ and Cy3 (coloured red and green; Dianova, Hamburg, Germany). Representative retinal images were captured at a distance of approximately $0.8-1 \mathrm{~mm}$ from the optic nerve head using a BH-2 microscope (Olympus, Melville, NY, USA).

Densitometric and statistical analysis Densitometry was analysed using SigmaGel 1.0 (Jandel Scientific, Erkrath, Germany) and SigmaPlot 4.0 (SPSS, Chicago, IL, USA) software. Data are presented as means \pm SD. A MannWhitney $U$-test (SPSS) was used for non-parametric comparisons between the two groups. A $p$ value of less than 0.05 was considered statistically significant.

\section{Results}

Changed molecular levels of 14-3-3 $\zeta$ and PKC isoenzymes In the diabetic retina, mRNA transcripts and protein levels of 14-3-3 $\zeta$ were significantly reduced by over $20 \%$ and $50 \%$, respectively $(p<0.05, n=3$ and $n=5$; Figs. 1a, 2a), while those of the PKC isoenzymes were significantly increased 1.27 - to 3 -fold $(p<0.05, n=3$ and $n=5$; Figs. 1b, 2b).

Interaction between 14-3-3 $\zeta$ and PKC proteins Total PKC protein, as well as PKC $\beta$ and $\zeta$ isoenzymes, directly interacted with 14-3-3 $\zeta$ in the retinas, and the interaction significantly decreased after 6 weeks of diabetes (Fig. 3).

$P K C$ activity $\mathrm{PKC}$ activity in PKC immunoprecipitate was significantly up-regulated (1.5-fold) in the retina after 6
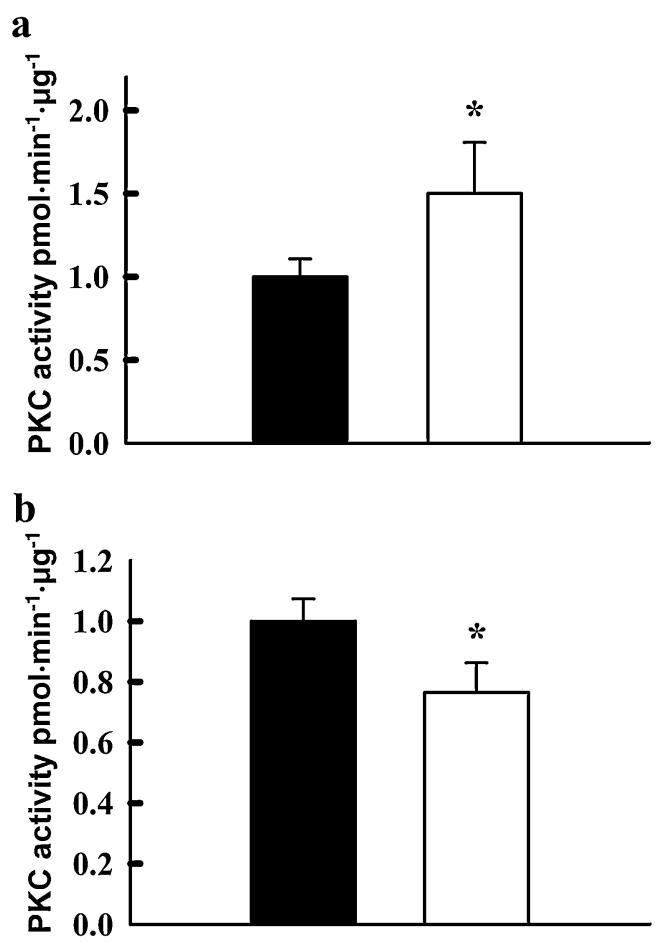

Fig. 4 PKC activity in PKC immunoprecipitates (a) and 14-3-3 $\zeta$ immunoprecipitates (b) in the retina after 6 weeks of diabetes vs control. PKC activity is presented as a fold change in diabetes (white bars) vs control (black bars). Data are means $\pm \mathrm{SD}, n=5$. ${ }^{*} p<0.05$ vs control 

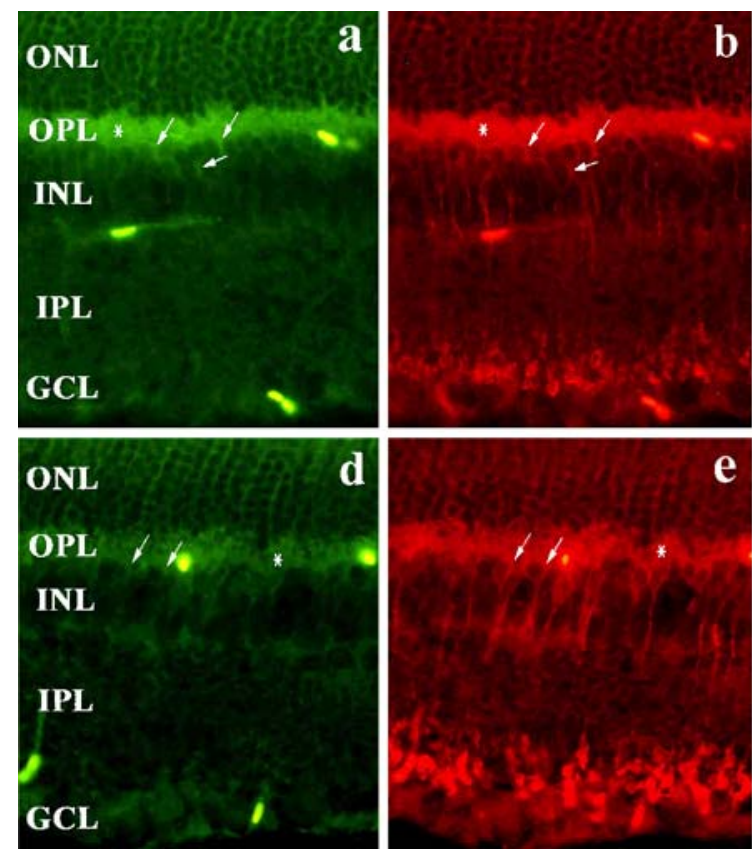

Fig. 5 Immunofluorescence staining for 14-3-3 $\zeta(\mathbf{a}, \mathbf{d})$ and PKC $(\mathbf{b}, \mathbf{e}, \mathbf{g})$ in the retina after 6 weeks of diabetes vs control $(\mathbf{a}-\mathbf{c}$ and d-g, respectively); $\mathbf{c}$ and $\mathbf{f}$ are superimposed images. $G C L$, ganglion cell layer; $I N L$, inner nuclear layer (small arrows); IPL, inner plex-

weeks of diabetes ( $p<0.05, n=3$; Fig. 4 a), while 14-3-3 $\zeta$ immunoprecipitate was inactivated ( $p<0.05, n=3$; Fig. $4 \mathrm{~b})$.

Co-localisation of 14-3-3 $\zeta$ and PKC proteins Figure 5 shows that 14-3-3 $\zeta$ and PKC were co-localised in the outer plexiform layer (asterisks) and at the membrane surface of some bipolar cells in the inner nuclear layer/inner plexiform layer margin (small and large arrows) in both control and diabetic retinas. The co-immunostained signals were markedly decreased in the diabetic retina (Fig. 5c, f). PKCpositive signals were often apparent in rod photoreceptors (arrowheads) of diabetic retinal sections (Fig. $5 \mathrm{~g}$ ).

\section{Discussion}

The isotype 1 14-3-3 $\zeta$ plays a critical role in neuronal differentiation, synaptic plasticity and exocytosis [1-6, 23], while PKC activation in diabetes implies diabetes-induced vascular dysfunction [14-16, 24]. In this study, the molecular levels of 14-3-3 $\zeta$ decreased in the retina after 6 weeks of diabetes, while those of PKC $\beta$ and $\zeta$ increased. This implies that the changes in 14-3-3 $\zeta$ and PKC levels might be associated with a disrupted balance between synaptic transmission and plasticity in the retina or with retinal dysfunction in early diabetes. Previously, we confirmed that isoforms other than 14-3-3 $\zeta$ did not show significant changes and that PKC $\beta$ and $\zeta$, among PKC isoenzymes, were remarkably up-regulated in the retina after 6 weeks of diabetes, although these data have not been published.

Many investigators have reported that $14-3-3 \mathrm{~s}$ act as potential regulators of $\mathrm{PKC}$, which is controlled by the

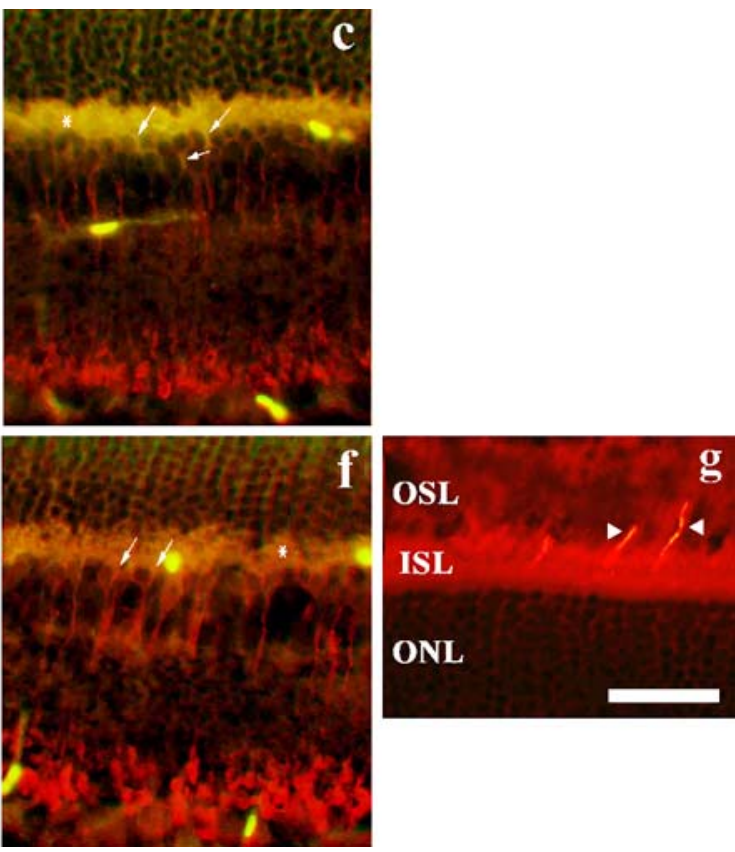

iform layer (large arrows); ISL, inner segment layer; $O N L$, outer nuclear layer; $O P L$, outer plexiform layer (*); $O S L$, outer segment layer. Bar represents $25 \mu \mathrm{m}$

interactions between them [10-13]. However, the effects of 14-3-3 on PKC have been controversial, showing conflicting results of inhibition or activation $[5,9,13]$, and the biological significance of this event remains unclear. In the present study, we sequentially confirmed decreased 14-3-3 $\zeta$ levels, up-regulated PKC and less interaction between the two in the diabetic retina. Also, PKC activity in PKC immunoprecipitate significantly increased in diabetes. The results suggest that the interaction between 14-3-3 $\zeta$ and PKC is negatively influenced by $14-3-3 \zeta$ expression rather than by PKC, and that the inhibitory action could be suppressed due to decreased $14-3-3 \zeta$ in the diabetic retina.

The decreased co-immunoreactivities of 14-3-3 $\zeta$ and PKC in the photoreceptor and bipolar cell dendrites of the diabetic retina, revealed in areas with high PKC activity, almost correspond to those for 14-3-3 $\zeta$, which is very important in considering the functional relationship between the two. Consequently, the decreased complex may induce a loss of signal transmission from photoreceptor terminals to bipolar cells.

In summary, a decrease in the 14-3-3 ¿/PKC complex may cause PKC activation and induce severe vascular or neuronal dysfunctions, as well as abnormal signal transduction in the retina, even in early diabetes. We suggest that regular expression of 14-3-3 $\zeta$ or of the 14-3-3 $\zeta / \mathrm{PKC}$ complex may be important in maintaining normal vision in the retina.

Acknowledgements This research was supported by a grant (M103KV01002-03K2201-02010) from the Brain Research Center of the 21st Century Frontier Research Program funded by the Ministry of Science and Technology, Republic of Korea. 


\section{References}

1. Hashiguchi M, Sobue K, Paudel HK (2000) 14-3-3zeta is an effector of tau protein phosphorylation. J Biol Chem 275: 25247-25254

2. Agarwal-Mawal A, Qureshi HY, Cafferty PW et al (2003) 14-33 connects glycogen synthase kinase-3 beta to tau within a brain microtubule-associated tau phosphorylation complex. J Biol Chem 278:12722-12728

3. Rubio MP, Geraghty KM, Wong BH et al (2004) 14-3-3-Affinity purification of over 200 human phosphoproteins reveals new links to regulation of cellular metabolism, proliferation and trafficking. Biochem J 379:395-408

4. Umahara T, Uchihara T, Tsuchiya $\mathrm{K}$ et al (2004) 14-3-3 proteins and zeta isoform containing neurofibrillary tangles in patients with Alzheimer's disease. Acta Neuropathol (Berl) 108:279-286

5. Gannon-Murakami L, Murakami K (2002) Selective association of protein kinase $\mathrm{C}$ with 14-3-3 zeta in neuronally differentiated PC12 cells. Stimulatory and inhibitory effect of 14-3-3 zeta in vivo. J Biol Chem 277:23116-23122

6. Martin H, Rostas J, Patel Y, Aitken A (1994) Subcellular localisation of 14-3-3 isoforms in rat brain using specific antibodies. J Neurochem 63:2259-2265

7. Kockel L, Vorbruggen G, Jackle H, Mlodzik M, Bohmann D (1997) Requirement for Drosophila 14-3-3 zeta in Raf-dependent photoreceptor development. Genes Dev 11:1140-1147

8. Rommel C, Radziwill G, Moelling K, Hafen E (1997) Negative regulation of Raf activity by binding of 14-3-3 to the amino terminus of Raf in vivo. Mech Dev 64:95-104

9. Van Der Hoeven PC, Van Der Wal JC, Ruurs P, Van Dijk MC, Van Blitterswijk J (2000) 14-3-3 isotypes facilitate coupling of protein kinase C-zeta to Raf-1: negative regulation by 14-3-3 phosphorylation. Biochem J 345:297-306

10. Van Der Hoeven PC, Van Der Wal JC, Ruurs P, Van Blitterswijk WJ (2000) Protein kinase $\mathrm{C}$ activation by acidic proteins including 14-3-3. Biochem J 347:781-785

11. Meller N, Liu YC, Collins TL et al (1996) Direct interaction between protein kinase $\mathrm{C}$ theta (PKC theta) and 14-3-3 tau in T cells: 14-3-3 overexpression results in inhibition of PKC theta translocation and function. Mol Cell Biol 16:5782-5791

12. Wheeler-Jones CP, Learmonth MP, Martin H, Aitken A (1996) Identification of 14-3-3 proteins in human platelets: effects of synthetic peptides on protein kinase $\mathrm{C}$ activation. Biochem $\mathrm{J}$ $315: 41-47$
13. Matto-Yelin M, Aitken A, Ravid S (1997) 14-3-3 inhibits the Dictyostelium myosin II heavy-chain-specific protein kinase C activity by a direct interaction: identification of the 14-3-3 binding domain. Mol Biol Cell 8:1889-1899

14. Aitken A, Howell S, Jones D et al (1995) Post-translationally modified 14-3-3 isoforms and inhibition of protein kinase C. Mol Cell Biochem 149-150:41-49

15. Birch KA, Heath WF, Hermeling RN et al (1996) LY290181, an inhibitor of diabetes-induced vascular dysfunction, blocks protein kinase $\mathrm{C}$-stimulated transcriptional activation through inhibition of transcription factor binding to a phorbol response element. Diabetes 45:642-650

16. Oku H, Kodama T, Sakagami K, Puro DG (2001) Diabetesinduced disruption of gap junction pathways within the retinal microvasculature. Invest Ophthalmol Vis Sci 42:1915-1920

17. Wigg SJ, Tare M, Forbes J et al (2004) Early vitamin E supplementation attenuates diabetes-associated vascular dysfunction and the rise in protein kinase C-beta in mesenteric artery and ameliorates wall stiffness in femoral artery of Wistar rats. Diabetologia 47:1038-1046

18. Kunisaki M, Bursell SE, Clermont AC et al (1995) Vitamin E prevents diabetes-induced abnormal retinal blood flow via the diacylglycerol-protein kinase C pathway. Am J Physiol 269: E239-E246

19. Bursell SE, Takagi C, Clermont AC et al (1997) Specific retinal diacylglycerol and protein kinase $\mathrm{C}$ beta isoform modulation mimics abnormal retinal hemodynamics in diabetic rats. Invest Ophthalmol Vis Sci 38:2711-2720

20. Koya D, King GL (1998) Protein kinase C activation and the development of diabetic complications. Diabetes 47:859-866

21. Kim YS, Kim YH, Cheon EW et al (2003) Retinal expression of clusterin in the streptozotocin-induced diabetic rat. Brain Res 976:53-59

22. Kim YH, Kim YS, Noh HS et al (2005) Change of rhodopsin kinase and transducin in the rat retina at early stage of diabetes. Exp Eye Res (in press)

23. Aitken A (1996) 14-3-3 and its possible role in co-ordinating multiple signalling pathways. Trends Cell Biol 6:341-347

24. Birch KA, Heath WF, Hermeling RN et al (1996) LY290181, an inhibitor of diabetes-induced vascular dysfunction, blocks protein kinase C-stimulated transcriptional activation through inhibition of transcription factor binding to a phorbol response element. Diabetes 45:642-650 